\title{
Endophthalmitis after removal of an encircling band
}

\author{
C E Hugkulstone, A S Rubasingham
}

\begin{abstract}
A case of endophthalmitis following the removal of an encircling band is presented. A possible route of entry in the absence of scleral perforation during the procedure is proposed.
\end{abstract}

After retinal detachment surgery, scleral buckles may need to be removed because of infection, erosion, extrusion, pain, ${ }^{1-4}$ or to reduce postoperative diplopia. ${ }^{5}$ The major complication associated with their removal is the risk of redetachment, with rates varying between $4 \%^{3}$ and $33 \%$.

We report a case of endophthalmitis following the removal of a scleral band.

\section{Case report}

A 36-year-old man was first seen in June 1988 with a subtotal retinal detachment in the left eye. He underwent an encircling procedure with a $2 \mathrm{~mm}$ silicone band secured at the equator with 5/0 Dacron sutures, with the sleeve in the superotemporal quadrant. Subretinal fluid was drained in the superotemporal quadrant as well. Postoperatively he was treated with Betnesol-N drops (betamethasone and neomycin) for six weeks.

Following surgery he complained of continuous pain in that eye, and he was admitted to hospital in March 1990 for release of the strap. He received no preoperative antibiotics.

Under general anaesthesia the conjunctiva was incised in three positions close to the strap site, and the fibrous capsule was dissected with blunt spring scissors. The strap was then divided and removed by gentle traction alone. The conjunctiva was closed with $6 / 0$ catgut, and chloramphenicol ointment was instilled. The following day the eye was settling satisfactorily and he was discharged home on twice daily chloramphenicol ointment.

Two days later he was readmitted with severe pain in the left eye and loss of vision. The visual acuity was perception of light. There was slight corneal epithelial oedema, a $2 \mathrm{~mm}$ hypopyon, and no red reflex could be elicited.

A pars plana vitreous tap was performed under local anaesthesia. Direct microscopy revealed a few leucocytes and Gram-positive cocci, and culture of the specimen grew Staphylococcus epidermidis.

He was treated with intracameral, subconjunctival, topical and systemic gentamicin, and systemic prednisolone was started after 48 hours. The endophthalmitis responded to treatment, and by seven weeks he had regained his pre- operative visual acuity of $6 / 36$. The retina has remained attached after four months' follow-up.

\section{Discussion}

At first sight this would appear to be a straightforward case of exogenous endophthalmitis. The classical organism was isolated ${ }^{6}$ and the onset was within 48 to 72 hours following surgery. However, the difficulty lies in the route of entry of the organism. There was no evidence of perforation of the globe during surgery, and no sutures were removed. No endogenous source for this infection was found.

It is possible that the site of the previous subretinal fluid drainage was disturbed by surgery, permitting direct entry of this normally commensal organism into the globe. This may have been aggravated by the scleral thinning that can occur beneath an encircling band, ${ }^{4}$ though there was no evidence of obvious scleral erosion in our patient.

Previous reports of the removal of scleral buckling elements have naturally concentrated on the risks of redetachment. ${ }^{1-4}$ However, one paper $^{3}$ does record a single case with intraocular exudate noted over the surface of the buckle, which resolved on removal of the buckle and administration of systemic antibiotics and steroids (though no vitreous tap was performed). There is also a solitary case from another series ${ }^{4}$ in which phthisis occurred from 'unexplained chronic intraocular inflammation' following removal of a scleral buckle. But there is no mention of proved postoperative endophthalmitis as a possible complication, despite some of these explants being removed because of infection. ${ }^{1-4}$ This may be because the majority of infected buckles are removed somewhat sooner than in our case, ${ }^{1-3}$ so reducing the extent of scleral thinning.

Although a single case report provides only weak grounds for any recommendations, the use of subconjunctival antibiotics may be prudent after the removal of scleral buckles, especially if previous drainage of subretinal fluid has been performed.

1 Ulrich RA, Burton TC. Infections following scleral buckling procedures. Arch Ophthalmol 1974; 92: 213-5.

2 Schwartz PL, Pruett RC. Factors influencing retinal redetachment after removal of buckling elements. Arch Ophthalmol 1977; 95: 804-7.

3 Hilton GF, Wallyn RH. The removal of scleral buckles. Arch Ophthalmol 1978; 96: 2061-3.

4 Lindsey PS, Pierce LH, Welch RB. Removal of scleral buckling elements. Causes and complications. Arch Ophthalmol 1983; 101: $570-3$

5 Fison PN, Chignell AH. Diplopia after retinal detachment surgery. Brf Ophthalmol 1987; 71: 521-5.

6 Diamond JG. Intraocular management of endophthalmitis. A systematic approach. Arch Ophthalmol 1981; 99: 96-9. 